\title{
EFFECT OF ACETYLCHOLINE ON THE CONTRACTILITY OF THE VAS DEFERENS AND EPIDIDYMIS AND ON FERTILITY OF MALE RATS
}

\author{
W. D. RATNASOORIYA \\ Department of Zoology, University of Colombo, Colombo 3, Sri Lanka.
}

(Date of receipt : 22.04.85)

(Date of acceptance : 01.08.86)

\begin{abstract}
Acetylcholine, a cholinoceptor agonist drug induced rhythmic contractions in isolated vasa deferentia and cauda epididymal tubules of rat. When the drug was administered chronically as a silastic formulation either to vas deferens (using $25 \%$ or $50 \%$ collars) or epididymis (using $50 \%$ rods) of rats, $50 \%$ rods significantly reduced spermatozon content in the ejaculate as well as in the cauda epididymis. $50 \%$ collars were toxic resulting in death within 2 hours. $25 \%$ collars failed to induce any significant alteration in spermatozoan distribution within the genital tract nor in ejaculatory spermatozoan count. Libido and fertility were not suppressed by both collars (25\%) and rods (50\%).
\end{abstract}

\section{Introduction}

In mammals, the cauda epididymis ${ }^{3,8,17}$ and the vas deferens ${ }^{3,9,15}$ are organs specialized for the storage of mature spermatozoa in a viable state until ejaculated either spontaneously or voluntarily. However, the storage capacity of these organs differ widely among the species studied. In general, the cauda appears to be the main storehouse ${ }^{1,15}$ of spermatozoa. The majority of spermatozoa present in the normal ejaculate emanate from the cauda epididymis. ${ }^{2}$. These observations suggest that ejaculates with low spermatozoan numbers would result if the spermatozoan reserves in the cauda epididymis and vasa deferentia are depleted considerably. If this is so, the fertility is likely to be suppressed as ejaculated spermatozoan numbers and fertility are closely related. 4,14 One possible way of achieving such a suppression in fertility via depletion of spermatozoan stores in cauda epididymis would be to induce rhythmic contractions in the caudae epididymides and/or vasa deferentia.

The present study was launched to investigate the above possibility in rats using acetylcholine, a cholinomimetic drug 24 and a method of local administration using medical grade Silastic elastomer. A cholinergic innervation has been demonstrated in the vas deferens ${ }^{10}$ and in cauda epididymis 6,10 and acetylcholine which is the neurotransmittor at the 
neuroeffector junctions of this innervation ${ }^{10}$ has been shown to induce rhythmic contractions of the epididymis in rats both in vitro ${ }^{13}$ and in vivo. ${ }^{18}$

\section{Experimental}

2.1 Drugs : Acetylcholine chloride (Sigma Chemical Co.) was used.

2.2 Animals : Healthy adult Sprague-Dawly rats of proven fertility (males weighing $200 \mathrm{~g}-250 \mathrm{~g}$ and females weighing $175 \mathrm{~g}-200 \mathrm{~g}$ ) were used. They were housed under the natural photoperiod of approximately $12 \mathrm{~h}$ light $/ 24 \mathrm{~h}$ at a constant temperature $\left(28^{\circ} \mathrm{C}-30^{\circ} \mathrm{C}\right)$ with free access to food (rat pellets, Moosajees Ltd., and green leaves) and tap water.

2.3 Preparation of sustained-released drug delivery systems : Silastic formulations containing $25 \%$ or $50 \%$ acetylcholine were constructed in the form of rods and collars by mixing known weights of drug and polysiloxane polymer (Silastic 382 Medical Grade Elastomer : Dow Corning Ltd, Midland, MI, U.S.A.) using a pestle and mortar as described in detail elsewhere. 20 Completed rods were $3.5 \mathrm{~mm}$ in diameter and $10-12 \mathrm{~mm}$ long and the collars were $8-10 \mathrm{~mm}$ long, $8 \mathrm{~mm}$ in diameter with a central cavity of about $2.8 \mathrm{~mm}$. A longitudinal slit was made along the wall of the collar to permit insertion around the vas deferens. Control rods and collars consisting only of Silastic were also made. The amount of acetylcholine present in $\mathrm{mg}$ in silastic formulations were (mean \pm s.e.m.) : $25 \%$ collars $80 \pm 7 ; 50 \%$ collars $160 \pm 10 ; 25 \%$ rods $24 \pm 0.6$; and $50 \%$ rods $49 \pm 1.2$.

2.4 Insertion of rods adjacent to epididymis and collars around the vas deferens : A single rod, or a collar was placed adjacent to each epididymis, or around each vas deferens at the prostatic end using aseptic precautions and mild ether anaesthesia as described in detail elsewhere. 20,21 . The day of insertion of the device was designated as Day $\mathrm{O}$. The numbers of rats used in each treatment group were : $50 \%$ acetylcholine rods 11 , control rods $8,50 \%$ acetylcholine collars $6,25 \%$ acetylcholine collars 6 , and control collars 8 .

2.5 Assessment of fertility : Libido and fertility of the rats fitted with $25 \%$ acetylcholine collars, $50 \%$ acetylcholine rods, and control rods and control collars were tested on days 3 and 7 , and then approximately at weekly intervals by pairing a pro-oestrous female having a regular 4-day vaginal cycle. Courtship and precopulatory sexual behaviour of the paired rats were observed up to 3 - 4 h from pairing. Insemination was confirmed either if there were spermatozoa in the oestrous vaginal smear on the following morning $(7.00-8.00 \mathrm{~h})$ or if the female went into prolonged vaginal dioestrous. At $9-12$ days Post coitum, the females were laparotomized and the number of embryos present in each uterine horn was recorded. For every group of treated males, a fertility index was calculated for each time interval 
following the insertion of rods or collars. The fertility index = total number of embryos present at laparatomy - number of successful matings at pairing.

2.6 Ejaculated spermatozoan count : A 50\% acetylcholine rod (10 rats) or a control rod (5 rats) was placed adjacent to each epididymis and a $25 \%$ acetylcholine collar ( 8 rats) or a control collar (5 rats) was placed around each vas deferens. Each of these rats was paired with a pro-oestrous female on days 3 and 7 respectively. On the following morning $(7.00-8.00 \mathrm{~h})$ vaginal smears were taken to check insemination and the spermatozoan numbers in the entire reproductive tract were estimated using a method described elsewhere. ${ }^{19}$

2.7 Motility of epididymal spermatozoa : The motility of epididymal spermatozoa was assessed in 14 rats, each of which was fitted with a single $50 \%$ acetylcholine rod adjacent to right epididymis and a single control rod adjacent to the left epididymis. At day 3 (6 rats) and at day 7 ( 8 rats) the motility of the spermatozoa from the two epididymides was scored using a subjective scale from. 0 (immotile) to 5 (greatest motility ever observed) as described elsewhere. ${ }^{20}$

2.8 Accessory reproductive organ weights : 6 rats were fitted with one 50\% acetylcholine rod adjacent to each epididymis and another 6 with a control rod, adjacent to each of their epididymides. On day 7 , these rats were killed by ether, weighed and their testes, epididymides, vasa deferentia, seminal vesicles together with coagulatory glands and prostate were excised, blotted free of any blood and extracellular fluids and weighed seperately on a Metler analytical balance. The weights of these organs were expressed as a percentage of body weight.

2.9 Spermatozoan counts in the male reproductive tract : A single 50\% acetylcholine rod (6 rats) or a control rod ( 8 rats) was placed adjacent to each epididymis and a single $25 \%$ acetylcholine collar (6 rats) or a control collar $(6$ rats) was placed around each vas deferens. On day 3, these rats were anaesthetized with ether and their reproductive systems were exposed through pelvic and scrotal incisions as described elsewhere. ${ }^{20,21}$ The entire epididymis and the vas deferens were removed from each side and was placed on a Whatman filter paper (diameter $11.0 \mathrm{~cm}$ ). The vas deferens was then cut off from the cauda epididymis at the cauda/vas junction and its length was measured. Each vas deferens was cut in to an epididymal half and a prostatic half at approximately $50 \%$ of its entire length. The corpus epididymis together with the caput epididymis was separated from the cauda epididymis at the corpus/cauda junction. All the segments from both sides of the reproductive tract were placed separately in $3.5 \mathrm{~cm}$ diameter petridishes, cut into eight portions of approximately equal size, macerated in a glass homogenizer, diluted with isotonic saline $(0.9 \% \mathrm{NaCl}$ in distilled water) and the spermatozoan content of each of the segments was estimated in 
quadraplicate using an improved Seabaure haemocytometer.

2.10 Spermatozoan counts in the urinary bladder $: 3$ rats were fitted with a single $50 \%$ acetylcholine rod and 2 rats with a control rod adjacent to each epididymis. In another set, a $25 \%$ acetylcholine collar (3 rats) or a control collar ( 2 rats) was placed around each vas deferens. On day 3 , these rats were paired individually with a pro-oestrous female at $18.00-19.00 \mathrm{~h}$ in an experimental room which was dimly lit with a $25 \mathrm{~W}$ bulb. These paired rats were then observed continuously until ejaculation occured. Immediately following ejaculation the males were anaesthetized with ether, urinary bladder was exposed through a pelvic incision and the entire contents of urinary bladder were withdrawn using a $1 \mathrm{ml}$ disposable syringe. A drop of this urine was transferred to a microscopic slide and examined for any spermatozoa.

2.11 Organ bath experiments : 15 rats were anaesthetized with ether and their vasa deferentia and epididymides were removed and placed immediately in petridishes containing fresh oxygenated $\left(95 \% \mathrm{O}_{2} ; 5 \% \mathrm{CO}_{2}\right)$ physiological salt solution having the following composition: $(\mathrm{mM} / \mathrm{l}): \mathrm{Na}^{+}, 141$; $\mathrm{K}^{+}, 5.9 ; \mathrm{Ca}^{++}, 2.6 ; \mathrm{Mg}^{++}, 1.2 ; \mathrm{Cl}^{-}, 104.8 ; \mathrm{H}_{2} \mathrm{PO}_{4}^{-}, 2.3 ; \mathrm{HCO}_{3}^{-}, 24.9$; $\mathrm{SO}_{4}^{--}, 1.2$ and glucose 11 . The vasa deferentia (length $29.5 \pm 1.5 \mathrm{~mm}$; mean \pm s.e.m, $N=8$ ) were then dissected free from the epididymides. A small section of the cauda epididymides (length $11.7 \pm 0.36 \mathrm{~mm}, \mathrm{~N}^{-}=18$ ) was uncoiled and freed from the surrounding connective tissue under a magnifying lens using a fine pair of forceps and entomological pins. The tissues were suspended in $50 \mathrm{ml}$ organ baths at $37 \pm 1^{\circ} \mathrm{C}$ under a resting tension of 1.0g. After an equilibration period of $20-30 \mathrm{~min}$ acetylcholine dissolved in isotonic saline was added cumulatively to the organ bath at $10-15 \mathrm{~min}$ intervals. Contractions were recorded isometrically with Dynamometer UF 1 Strain gauges using Washington 400 MD 2 polygraph.

2.12 Release rate from $50 \%$ acetylcholine rods : A weighed $50 \%$ acetylcholine rod was fitted adjacent to each epididymis of 8 rats. On day 7, these rods were removed under ether anaesthesia blotted free of body fluids and dried at $60^{\circ} \mathrm{C}$, until a constant weight was recorded. The amount of acetylcholine released was estimated by substracting the final weight from the initial weight.

\section{Results}

The acetylcholine treatment caused marked stimulation of salivary and lachrymal secretions, and exopthalmia and constriction of pupils even before the surgery was completed indicating the release of drug from these silastic devices as these are characteristic actions reported with cholinomimetic drug administration. ${ }^{23}$ In addition, bleeding from the eyes and voiding of urine 
epididymis. Furthermore, rhythmic contractions in the cauda epididymis in vivo has been reported with methoxamine when administered locally, using a drug delivery system identical to that used in this study. ${ }^{20}$ It is likely that these contractions may have propelled the spermatozoa from the cauda epididymides into the vasa deferentia, since there was a slight, but nonsignificant rise in spermatozoan numbers within it. Since this increase in spermatozoan number in the vas was not significant it is presumed that the expelled spermatozoa from the cauda epididymides were eventually voided in urine $e^{7,25}$ or spontaneously ejaculated. ${ }^{1,16}$ Alternatively, a reduction in spermatozoan population in the cauda epididymis could have resulted from an increase in output of lytic enzymes thereby digesting the spermatozoa found within it as lytic enzymes ${ }^{12}$ and dissolution of spermatozoa ${ }^{5}$ have been demonstrated in cauda epididymides. Furthermore, there is evidence to suggest that the cholinergic neurones in the vas deferens and in the epididymis are secretomotor. ${ }^{22}$ Further experiments are however needed to test these possibilities.

$50 \%$ acetylcholine rods but not $25 \%$ acetylcholine collars produced oligospermic ejaculates. Such oligospermic ejaculates may have resulted from retrograde ejaculation, as it is known that stimulation of parasympathetic neurones causes relaxation of the internal sphincter of the urinary bladder. ${ }^{10}$ But this can be ruled out since $25 \%$ collars which were placed close to the bladder failed to induce oligospermia and because no spermatozoa were detected in the urinary bladder following mating in rats fitted with $25 \%$ acetylcholine collars and or with $50 \%$ acetylcholine rods. Dysfunctioning of the ejaculatory mechanism is also unlikely in view of the role played by the cholinergic mechanism in the production of emission and ejaculation ${ }^{23}$ and considering the pharmacology of the drug. ${ }^{24}$

$50 \%$ acetylcholine collars produced toxic effects. Such effects were also evident with $25 \%$ collars and $50 \%$ rods but with reduced intensity. The differences in the intensities of toxicity between $50 \%$ collars and $50 \%$ rods may be attributed to their respective placements within the body ; collars in abdominal cavity and rods in scrotal sacs. Libido and fertility were not suppressed by $25 \%$ collars and $50 \%$ rods. A suppression in libido is not reported with cholinomimetic drugs. ${ }^{24}$ The unimpairment of fertility despite oligospermia indicates that the suppression in spermatozoan numbers in the ejaculate is not sufficiently large to evoke sterility. However, this could have been possible with rods if the drug concentration had been increased further. But this is not feasible using the current drug-delivery system. ${ }^{2.6}$ However, it seems worthwhile to continue these studies with other therapeutically used potent cholinomimetic drugs having lesser side effects, as this study demonstrates that it is possible to induce oligospermia. with acetylcholine rods without simultaneous loss of libido. 


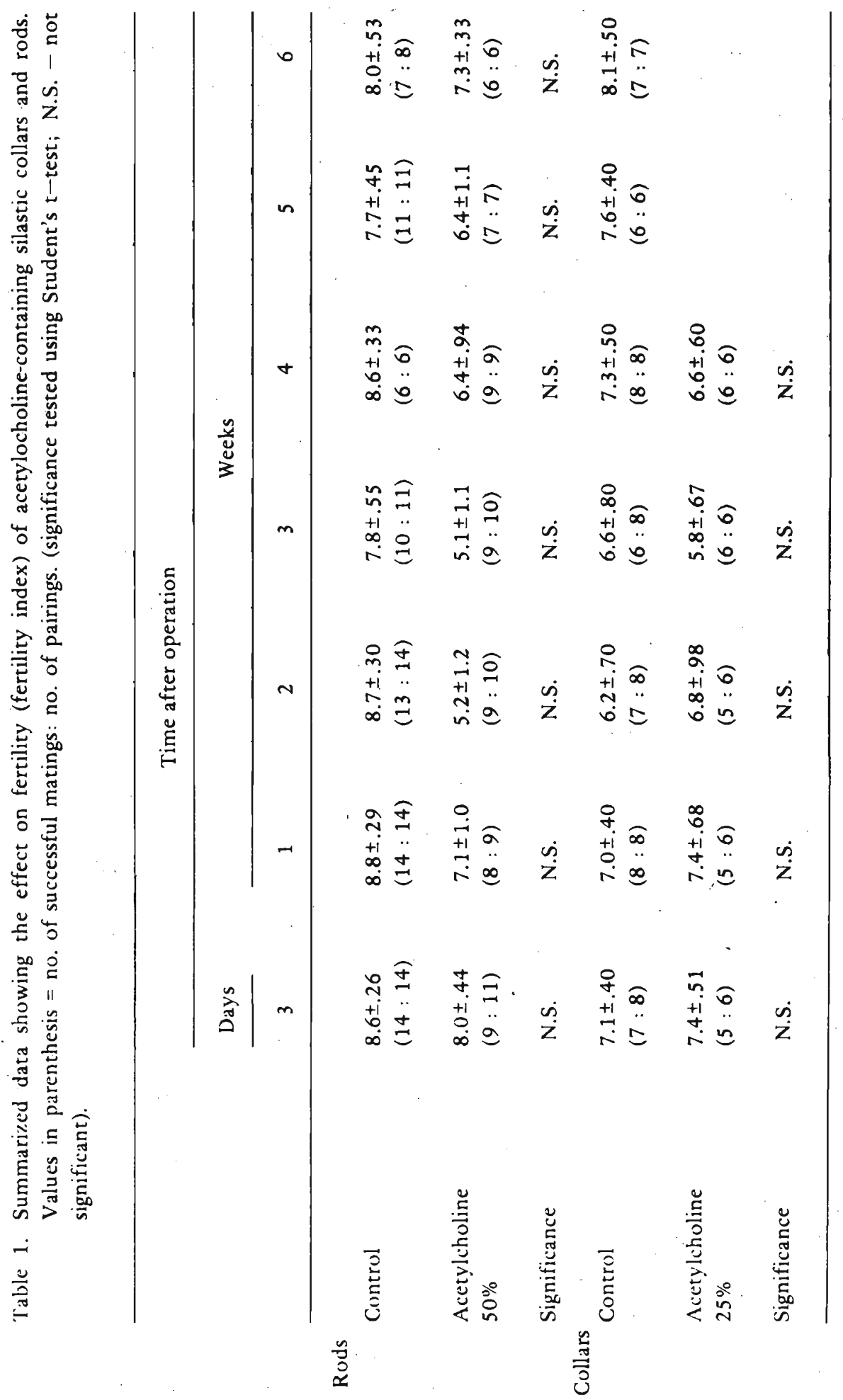




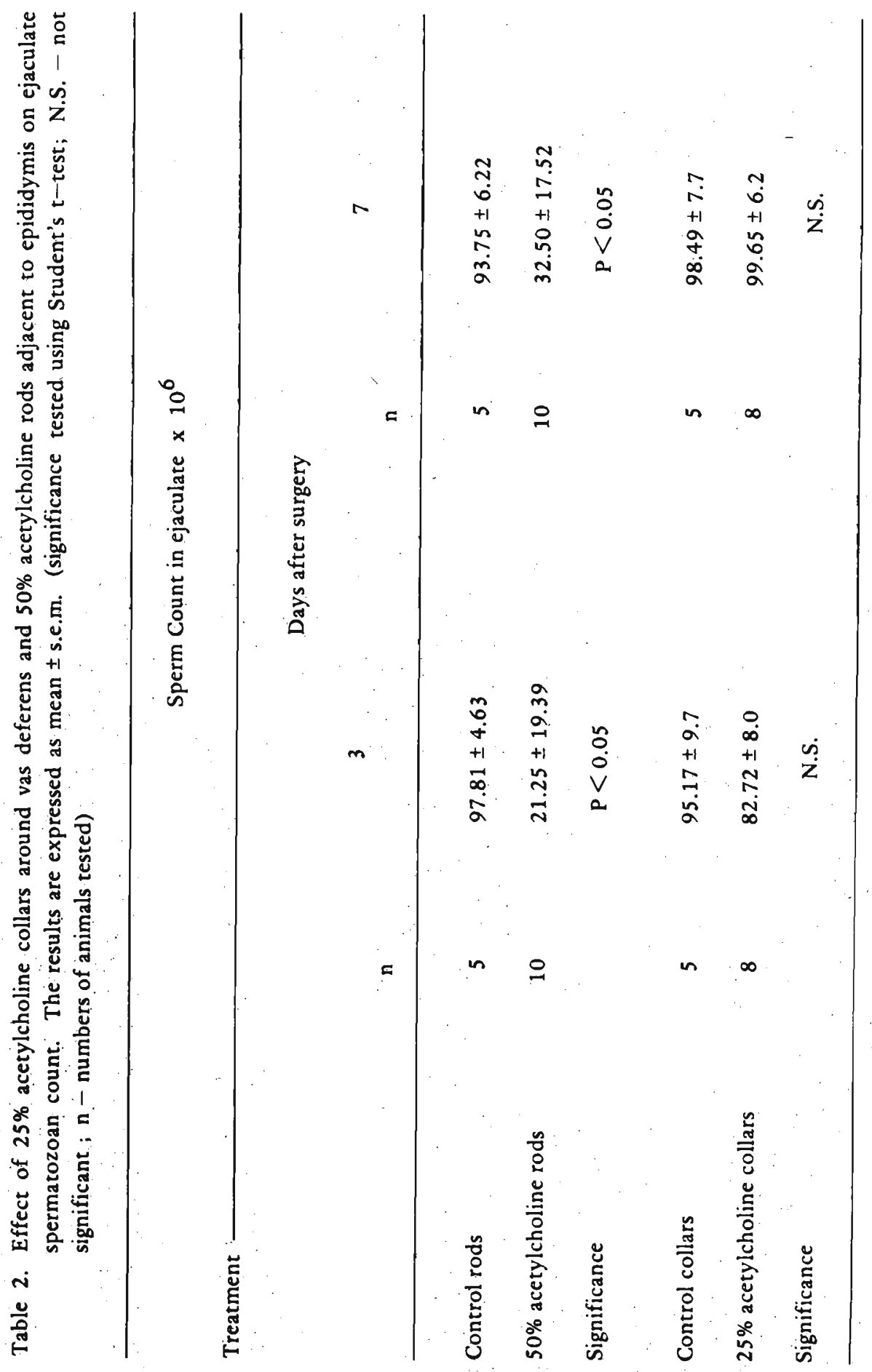




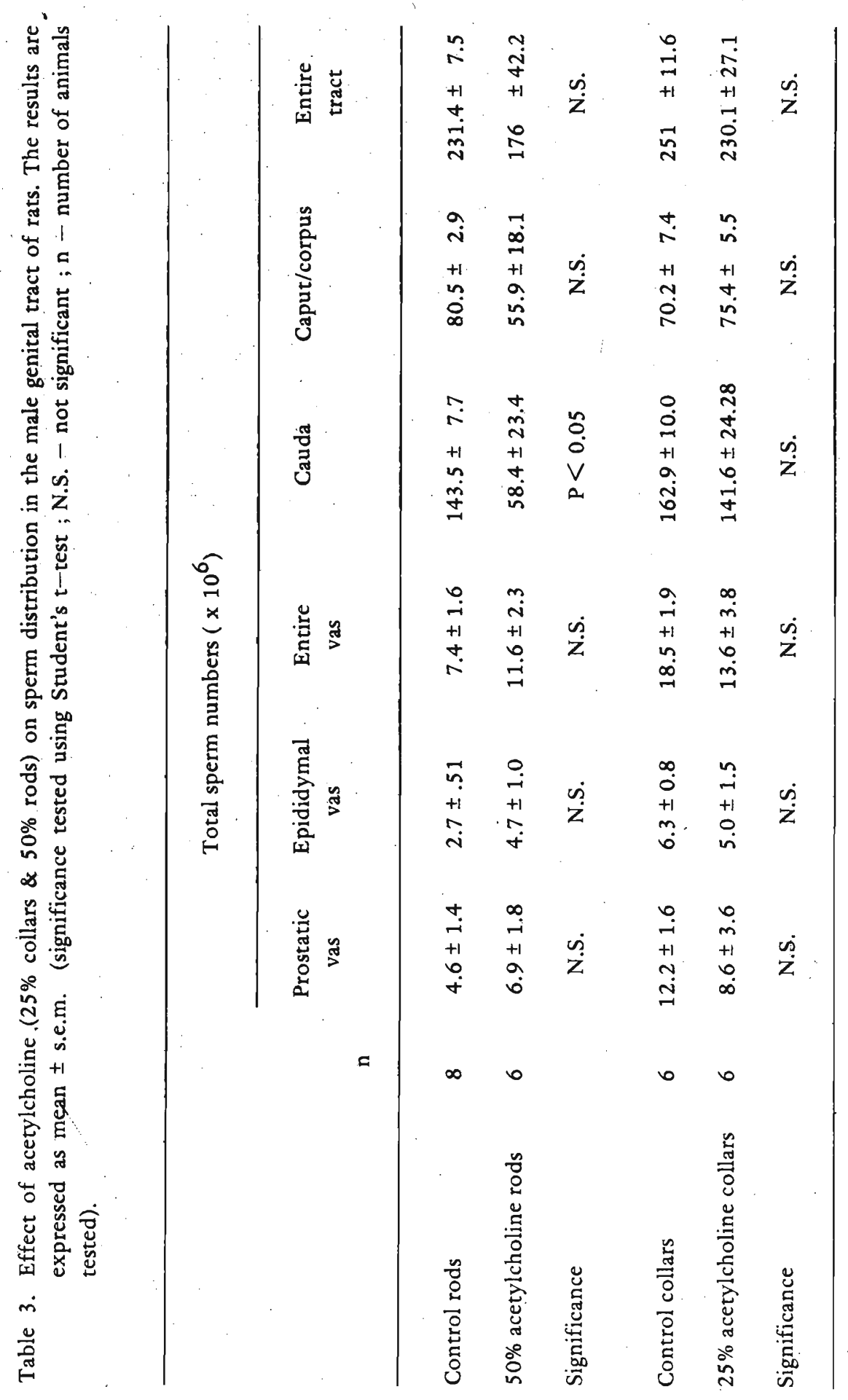


were evident with $50 \%$ collar treatment. All the rats in this treatment group died between $10-120 \mathrm{~min}$ following the completion of surgery. In contrast, no deaths were seen with $50 \%$ acetylcholine rods or with $25 \%$ acetylcholine collars. The food and water intake, and the general appearance and behaviour of these rats were similar to the rats in the control group.

3.1 Assessment of fertility : Acetylcholine treatment (25\% collars and 50\% rods) did not cause any apparent alteration in libido as judged by undiminished courtship and precopulatory sexual behaviour. Furthermore, this treatment did not significantly impair the mating performance as judged by percentage of successful matings $(P>0.05$; Fisher Exact test $)$ - the percentages being $96 ; 92 ; 92$ and 90 with control rods, control collars, $50 \%$ acetylcholine rods and $25 \%$ acetylcholine collars, respectively.

The results of the fertility experiments are summarized in Table 1 and it can be seen that the fertility of rats treated with acetylcholine was not significantly impaired. ( $P>0.05$; Student's t-test).

3.2 Ejaculated spermatozoan count : The results of the ejaculate spermatozoan counts on days 3 and 7 are summarized in Table 2. A reduction in mean ejaculate spermatozoan counts was evident in rats fitted with $50 \%$ acetylcholine rods adjacent to their epididymides ( $\mathrm{P}<0.05$; Student's $t$-test).

3.3 Motility of epididymal spermatozoa : The average motility scores of spermatozoa on the control side on days 3 and 7 were (mean \pm s.e.m.) $4.0 \pm 0.2$ and $4.2 \pm 0.4$, and those on the treated side were $3.8 \pm 0.4$ and $3.6 \pm 0.2$, respectively - the drug exerting no significant effect on motility (P>0.05; Mann-Whitney U-test).

3.4 Accessory organ weights : The average weights $(\mathrm{mg} / 100 \mathrm{~g}$ body weight \pm s.e.m) of testes, epididymides, vasa deferentia, seminal vesicular-coagulatory gland complex and prostate of rats with control rods were 1051 \pm 49 , $385 \pm 05,67 \pm 06,673 \pm 49$ and $186 \pm 13$ respectively, while in rats fitted with $50 \%$ acetylcholine rods the corresponding values were $1205 \pm 42$, $391 \pm 18,72 \pm 04,445 \pm 78$ and $133 \pm 17$. These differences in weights of the organs between the two groups are not significant. $(P>0.05$; Student's t-test).

3.5 Spermatozoan counts in the male reproductive tract : The results of the spermatozoan distribution are summarized in Table 3. Application of $25 \%$ collars did not cause a significant change in spermatozoan distribution in any part of the tract $(\mathrm{P}>0.05$; Student's t-test). In contrast, $50 \%$ rods have brought about a significant $(\mathrm{P}<0.05)$ reduction in spermatozoan numbers in the cauda epididymis. 


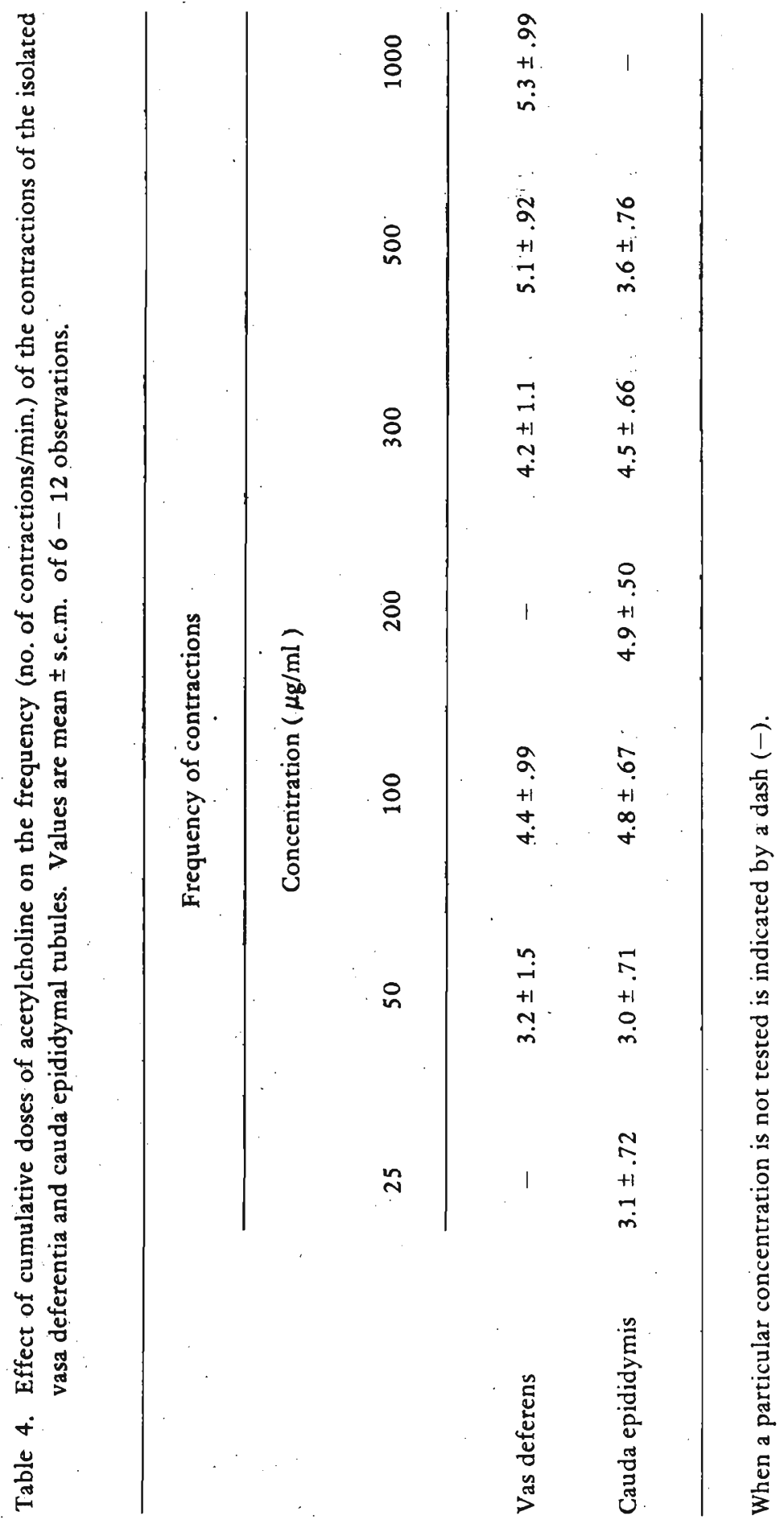


3.6 Spermatozoan counts in the urinary bladder : No spermatozoa were detected in the urine samples of any of the rats investigated.

3.7 Organ bath experiments : Neither the vasa deferentia nor the epididymal tubules exhibited spontaneous contractions during the period of equilibration. However, the addition of acetylcholine elicited rhythmic contractions in both tissues. The frequencies of these contractions at the concentrations tested are given in Table 4 . The amplitude of these contractions in the vas ranged between 0.01 to $0.22 \mathrm{~g}$ and in the epididymis between $0.05 \mathrm{~g}$ to $0.70 \mathrm{~g}$.

3.8 Release rate from $50 \%$ acetylcholine rods : The average release rate of acetylcholine from $50 \%$ rods was $10.6 \pm 1.4 \mathrm{mg}$ after 7 days.

\section{Discussion}

In this study no spontaneous contractions were recorded in isolated vasa deferentia and in cauda epididymal tubules. In the rat, the vas deferens is normally quiescent unlike the cauda epididymal tubules which show spontaneous contractions. ${ }^{13}$ The differences in ionic composition of the physiological salt solutions and in resting tensions used in the present study and in that of Laitinen and Talo ${ }^{13}$ may have been responsible for the lack of spontaneous contractions observed in the cauda epididymal tubules.

Several workers have demonstrated that rhythmic contractions can be induced with acetylcholine, or cholinoceptor agonist drug, ${ }^{24}$ in cauda epididymal tubules both in vivo ${ }^{18}$ and in vitro. ${ }^{11,13}$. The results of this study confirm these previous data ${ }^{11,13}$ and also show that similar rhythmic contractions can be initiated in isolated vasa deferentia as well.

In the epididymis, atropine, a cholinoceptor antagonist drug ${ }^{24}$ has been shown to abolish contractions induced by carbachol, ${ }^{\mathrm{P}}$ a cholinoceptor agonist drug ${ }^{24}$ suggesting that these contractile responses are mediated via cholinoceptors found among the smooth muscles of the tubules. As a fairly dense cholinergic innervation is present in the vas deferens ${ }^{10}$ as it is in the epididymis 6,10 it is likely that these contractions evident with acetylcholine in the vas deferens are also mediated via cholinoceptors which are present in association with the cholinergic 'innervation in the vas. There is no clear evidence from this study to show that the rhythmic contractions observed in vitro in isolated vas deferens and in cauda epididymal tubules were also produced in situ when collars (around vas) and rods (adjacent to epididymis) containing acetylcholine were implanted. However, the fact that a significant reduction in spermatozoan numbers were seen in cauda epididymis subsequent to the implantation of acetylcholine rods but not with acetylcholine collars suggests that contractions might have occured at least in the 


\section{Acknowledgements}

Thanks are due to Dr. S.U.K. Ekaratne, Department of Zoology, University of Colombo for critically reading the manuscript. This work was supported by W.H.O. (Grant No: 79034) and the Natural Resources, Energy and Science Authority of Sri Lanka (Grant No: 83/44).

\section{References}

1. AGMO, A. (1976) J. Reprod. \& Fert. $48: 405-407$.

2. ALMQUIST, J.O. \& AMANN, R.P. (1961) J. Dairy Sci., 44 : 1668-1678.

3. AMANN, R.P., JOHNSON, L., THOMPSON, D.L. \& PICKETT, B.W. (1976) Bio. Reprod. 15 : 586-592.

4. BARNEA, E.R., ARRONET, G.H., WEISSENBERG, R. \& LUNENFELD, B. (1980) Int. J. Fert., 25(4) : 303-306.

5. COOPER, T.G. \& HAMILTON, D.W. (1977) Am. J. Anat., 149(1) : 93-110.

6. EL-BADAWI, A. \& SCHENK (1962) Am. J. Anat.; $121: 1-14$.

7. FERNANDEZ-COLLAZO, E., VIDELA, E. \& PEREYRE, J.C. (1971) J. Reprod. \& Fert. $27: 145-147$.

8. FELDESY, P.G. \& BEDFORD, J.M. (1982) Biol. Reprod., $26: 673-682$.

9. FREUND, M. \& DEVIS, J. (1969) Fert. Steril., $20: 163-170$.

10. GANONG, W.F. (1977) In review of Medical Pbysiology, pp. 313-344., Japan : Large Medical Publications.

11. HIB, J. \& CALDEYRO-BARCIA, R. (1974) In Pbysiology and Genetics of Reproduction. pp. 111-126, Plenum Press, New York.

12. JONES, R. (1974) J. Endocr.6 7: 157-165.

13. LAITINEN, L. \& TALO, A. (1981) J. Reprod. Fert. .6 3: 205-209.

14. MACLEOD, J. (1973) Hosp. Pract., 8 : 43-52.

15. NEAVES, W.B. (1975) In Handbook of Pbysiology, 7(5) : pp. 383-404, Baltimore : Williams \& Willkins. 
16. ORBACH, J. (1961) Science, N.Y. $134: 1072-1073$.

17. ORGEBIN-CRIST (1969) Biol. Reprod., 1 : 155-175.

18. PHOLPRAMOOL, C. \& TRIPHRON,.N. (1984) J. Reprod. Fert., $71: 181-188$.

19. RATNASOORIYA, W.D. (1979) J. Pbarmaco. Methods. $2: 379-381$.

20. RATNASOORIYA, W.D., GILMORE, D.P. \& WADSWORTH, R.M. (1984) J. Reprod. Fert. 58 : 19-25.

21. RATNASOORIYA, W.D., GILMORE, D.P \& WADSWORTH, R.M. (1979) J. Reprod. Fert. $56: 643-651$.

22. SJOSTRAND, N.O. \& KLINGE, E. (1977). Acta. Pbysiol. Scand. Suppl. 452 : 89-91.

23. THOMAS, A.J. (1983) Fertil. Steril., 39(4) : 445-454.

24. TURNER, P: \& RICHENS, A. (1973): In Clinical Pbarmacology, pp. 25-53, Edinburgh \& London: Churchill Livingstone.

25. UREEBURG, J.T.H., VAN ANDEL, M.V., KORT, W.J. \& WESTBROCK, D.L. (1974). J. Reprod. Fert., 41 : 355-359.

26. WADSWORTH, R.M. \& RATNASOORIYA, W.D. (1981). J. Pbarmacol. Metbods., $5: 313-320$. 